\title{
Effect of Locally Delivered Minocycline Microspheres on Markers of Bone Resorption
}

\author{
R.J. Oringer, ${ }^{*}$ K.F. Al-Shammari, ${ }^{\dagger}$ W.A. Aldredge, ${ }^{*}$ V.J. Iacono, ${ }^{*}$ R.M. Eber, ${ }^{\dagger}$ H.-L. Wang, ${ }^{\dagger}$ B. Berwald, ${ }^{*}$ \\ R. Nejat, ${ }^{*}$ and W.V. Giannobile ${ }^{\dagger}$
}

Background: Gingival crevicular fluid (GCF) biomarkers associated with bone resorption may be useful to determine periodontal disease status and response to therapy. The pyridinoline cross-linked carboxy-terminal telopeptide of type I collagen (ICTP), a bone-specific degradation product, and interleukin 1-beta (IL1), a potent bone-resorptive cytokine, have both been associated with periodontal disease activity. Minocycline is a tetracycline derivative possessing antimicrobial effects on periodontal pathogens and inhibitory properties on matrix metalloproteinases (MMPs) associated with tissue destruction. The aim of this study was to evaluate the effect of periodontal treatment in the form of scaling and root planing (SRP) and locally administered minocycline microspheres on the GCF levels of ICTP and IL-1.

Methods: Forty-eight chronic periodontitis patients were randomly assigned to 2 groups (SRP plus subgingival application of vehicle control [SRP + V], or SRP plus subgingival application of minocycline microspheres [SRP $+M]$ ) and monitored at 8 sites per subject at baseline and 1, 3, and 6 months. Four shallow $(P D \leq 3 \mathrm{~mm}$ ) and 4 deep (PD $\geq 5 \mathrm{~mm}$ ) sites were evaluated for both marker levels and for probing depth (PD), clinical attachment level (CAL), and bleeding on probing (BOP). Eight periodontally healthy control subjects with no probing depths $>3 \mathrm{~mm}$ and no loss of attachment were also monitored at the same time intervals. GCF levels of ICTP and IL-1 were determined using radioimmunoassay and enzyme-linked immunosorbent assay techniques, respectively.

Results: Significant differences $(P<0.001)$ in GCF levels of ICTP and IL-1 were found between deep and shallow sites at all time points in both treatment groups. In addition, healthy subjects demonstrated significantly reduced levels of both markers compared to both shallow and deep sites in periodontitis patients $(P<0.001)$. Only the SRP $+M$ treated patients exhibited significant reductions $(P<0.05)$ in both ICTP and IL-1 levels 1 month after treatment. Furthermore, the SRP + M group demonstrated significantly lower IL-1 levels $(P<0.02)$ at 1 month compared to the SRP $+\mathrm{V}$ group.

Conclusions: Results of this study indicate that GCF levels of ICTP and IL-1 correlate with clinical measures of periodontal disease and may aid in assessing disease status and response to periodontal therapy. Furthermore, local administration of minocycline microspheres led to a potent short-term reduction in GCF IL-1 levels. Additional studies are needed to address whether repeated administration of scaling and root planing along with minocycline microspheres will achieve long-term reductions in GCF ICTP and IL-1 levels. J Periodontol 2002;73:835-842.

\section{KEY WORDS}

Bone resorption; clinical trials; drug delivery systems; interleukin-1; periodontal diseases/therapy; pyridinoline cross-links; collagen.

\footnotetext{
* Department of Periodontics, School of Dental Medicine, Stony Brook University, Stony Brook, NY.

$\dagger$ Department of Periodontics/Prevention/Geriatrics, School of Dentistry, University of Michigan, Ann Arbor, MI.

‡ Center for the Biorestoration of Oral Health, School of Dentistry, University of Michigan.
} 
$\mathrm{P}$ eriodontal diagnostic methods have traditionally relied on visual and physical assessments of gingival inflammation and periodontal tissue damage (e.g., gingival redness, bleeding on probing, probing depth and clinical attachment level measurements, and radiographic examination). ${ }^{1}$ These methods have proven effective as post hoc indicators of periodontal disease history; however, they are unable to identify highly susceptible patients or to distinguish between disease-active and inactive sites. ${ }^{2-4}$ Therefore, diagnostic tests that can detect the presence of active disease, predict future disease progression, and evaluate the response to periodontal therapy may prove valuable in improving the clinical management of periodontal patients. ${ }^{5-7}$

Gingival crevicular fluid (GCF) is a modified serum transudate or inflammatory exudate derived from the periodontal tissues that appears during altered states of vascular permeability associated with gingival inflammation. $3,8 \mathrm{GCF}$ constituents are derived from serum, host connective tissue and epithelium, inflammatory cells, and bacteria. ${ }^{8}$ Many GCF components have been evaluated for potential use as periodontal diagnostic aids. ${ }^{3,8}$ However, most of the investigated markers are not specific for alveolar bone destruction, and may therefore not distinguish between gingivitis and active periodontitis (i.e., progressive attachment or alveolar bone loss). ${ }^{9}$

Pyridinoline cross-links represent a class of collagen degradative molecules that include pyridinoline, deoxypyridinoline, $\mathrm{N}$-telopeptides, and C-telopeptides. ${ }^{10,11}$ Subsequent to osteoclastic bone resorption and collagen matrix degradation, pyridinoline, deoxypyridinoline, and amino- and carboxy-terminal cross-linked telopeptides of type I collagen (ICTP) are released into the circulation. Because the cross-links and cross-linked telopeptides result from post-translational modification of collagen molecules, they cannot be recycled during collagen synthesis, and are therefore considered specific for bone resorption. ${ }^{12}$ Elevated serum ICTP and other pyridinoline cross-link levels have been shown to correlate with the bone-resorptive rate in several osseous metabolic diseases, including osteoporosis, rheumatoid arthritis, and Paget's disease. ${ }^{13-15}$ Serum ICTP levels have also been used as prognostic indicators in several primary and metastatic bone tumors such as multiple myeloma and prostate cancer. $^{16,17}$

Several investigations have recently explored the ability of pyridinoline cross-links to detect bone resorption in periodontitis ${ }^{18-22}$ and peri-implantitis. ${ }^{23}$ Additionally, treatment with a matrix metalloproteinase (MMP) inhibitor significantly reduced GCF ICTP levels coincident with suppression of MMP-8 and MMP-13 in periodontitis patients. ${ }^{20}$ The results of these studies suggest that utilization of ICTP may be beneficial to identify and predict periodontal disease progression and monitor response to treatment.

Interleukin-1 (IL-1) is a potent bone-resorbing cytokine produced by a variety of cells including monocytes, neutrophils, fibroblasts, and keratinocytes. ${ }^{1,24}$ IL- $1 \beta$ is the major form secreted by monocytes, and its production is stimulated by bacterial lipopolysaccharide. ${ }^{25}$ Once secreted, IL-1 may activate lymphocytes, incite macrophage chemotaxis and prostaglandin production, and stimulate osteoclastic resorption of bone. ${ }^{26} \mathrm{IL}-1$ has been detected in gingival biopsies and in the GCF of periodontal patients. ${ }^{27-31}$ Cross-sectional studies have also shown increased GCF IL-1 levels in sites with periodontitis compared to healthy sites, ${ }^{30}$ and at sites with experimental gingivitis. ${ }^{32}$

Minocycline $\mathrm{HCl}$ is a tetracycline derivative that is active against a broad spectrum of Gram-negative and Gram-positive anaerobes including organisms implicated in chronic periodontitis. ${ }^{33}$ In addition, tetracycline derivatives such as minocycline inhibit MMPs that destroy constituents of the periodontium including collagen, elastic fibers, proteoglycans, and fibronectin. ${ }^{34-36}$ Recently, minocycline has been incorporated into a controlled-release bioabsorbable polymer (poly glycolide-co-dl-lactide) or PGLA, which serves as the encapsulating medium. This delivery system releases the antibiotic over a 2 -week period at the site of administration at concentrations exceeding 300 $\mu \mathrm{g} / \mathrm{ml}$ as measured in the GCF. ${ }^{37}$ A recent clinical trial demonstrated that utilization of this therapy§ significantly enhanced scaling and root planing (SRP) when compared to SRP alone. ${ }^{38}$

The aim of this investigation was to examine the effect of SRP and locally delivered minocycline microspheres on GCF levels of ICTP and IL-1 in patients with chronic periodontitis.

\section{MATERIALS AND METHODS}

\section{Subject Population}

Forty-eight patients with moderate to severe chronic periodontitis participated in this clinical trial. These patients were required to have at least 4 teeth with probing depth measurements of 6 to $9 \mathrm{~mm}$ that bled upon probing. Eligible subjects had not received any periodontal therapy for a minimum of 6 months and no antibiotic treatment for at least 3 months prior to the study, and were not chronically treated (i.e., 2 weeks or more) with any medication known to affect periodontal status (e.g., phenytoin, calcium antagonists, cyclosporin, coumadin, and non-steroidal anti-inflammatory drugs). In addition, 8 periodontally healthy control subjects exhibiting no attachment loss and no radiographic bone loss were also recruited. The study protocol was approved by the Institutional Review Boards

$\S$ Arestin, OraPharma, Inc., Warminster, PA. 
at both the University of Michigan and Stony Brook University, and informed consent was obtained in writing from each subject prior to study commencement. This was a randomized, single-masked, vehicle-controlled 6 -month study with 2 parallel arms. The study presented is a substudy of a multicenter investigation recently reported by Williams et al. ${ }^{38}$ In addition, results derived from an additional arm (SRP alone) have recently been published. ${ }^{18}$ The 2 arms of the present study were: 1) SRP plus subgingival application of minocycline microspheres $(\mathrm{SRP}+M)$, and 2) SRP plus subgingival application of vehicle (SRP $+\mathrm{V})$.

Collection of GCF was performed at baseline (prior to treatment), and at 1, 3, and 6 months. After the baseline procedures (i.e., GCF sampling and clinical measurements) were completed, SRP was performed using hand and ultrasonic instrumentation. A maximum of 2 sessions within 14 days with no time limitation was allowed to complete each subject. Local anesthetic was used at the discretion of the clinician. After SRP was completed, patients were stratified according to smoking status and randomly assigned to treatment groups. Smoking status was defined as positive or negative based upon whether the patient had used tobacco products within the last 6 months. Study treatments (minocycline microspheres or vehicle) were subgingivally applied at baseline to all sites with $P D \geq 5 \mathrm{~mm}$. Sites in the SRP $+M$ group were given a unit dose of $4 \mathrm{mg}$ containing $1 \mathrm{mg}$ of minocycline and $3 \mathrm{mg}$ of polymer, while sites in the $\mathrm{SRP}+\mathrm{V}$ group received $3 \mathrm{mg}$ of polymer only. Study treatments were reapplied at 3 and 6 months at all sites initially treated at baseline. The subjects in the periodontally healthy control group received a prophylaxis at baseline and no other treatment throughout the trial. An outline of the study design is summarized in Table 1.

\section{GCF Sampling}

For the periodontitis patients, each subject was monitored at 8 sites at baseline (prior to SRP), and 1, 3, and 6 months after treatment. Four shallow (PD $\leq 3$ $\mathrm{mm}$ at screening, no radiographic alveolar bone loss) and 4 deep (PD $\geq 5 \mathrm{~mm}$ at screening, $\geq 3 \mathrm{~mm}$ from cemento-enamel junction to bone crest) sites were monitored longitudinally in each subject for both marker levels (i.e., ICTP and IL-1). Only deep sites received subgingival application of minocycline microspheres or vehicle. ICTP and IL-1 levels were determined at different sites within each subject (i.e., 2 shallow/2 deep sites per mediator for a total of 8 sites per subject). For the periodontally healthy control subjects, GCF samples were collected from 4 randomly selected sites (no attachment loss, PD $\leq 3 \mathrm{~mm}$ ) at baseline and 1, 3, and 6 months. The area around each site sampled was dried and supragingival plaque removed. GCF was collected for 30 seconds using methylcellulose strips. Each strip was placed gently into the gingival crevice until slight resistance was felt, and GCF volume was determined. " Following collection and volume determination, the samples were kept on ice for transport to the laboratory and stored at $-20^{\circ} \mathrm{C}$.

ICTP. The laboratory procedures for analysis of ICTP samples were performed as described by Giannobile et al. ${ }^{19}$ The frozen samples were brought to room temperature, followed by elution of proteins by microcentrifugation. Levels of ICTP were determined using a radioimmunoassay technique ${ }^{\#}$ as previously described. ${ }^{39}$

IL-1. The IL- 1 content was determined by using a commercially available enzyme-linked immunosorbent (ELISA) assay** based on a double antibody sandwich technique described by Payne et al. ${ }^{40}$ Both ICTP and IL-1 were reported as total amounts per time of collection (pg/30-second sample). All analyses were performed with masking of the treatment groups maintained.

\section{Clinical Measurements}

Clinical measurements were completed at a total of 384 sites (i.e., 48 subjects $\times 8$ sites/subject) at baseline and 1, 3, and 6 months. Examiners were masked in regards to the treatment that each subject received. The parameters recorded included: 1) probing depth (PD), measured from the gingival margin to the apical penetration of the probe tip in millimeters; 2) clinical attachment level (CAL), measured from either the cemento-enamel junction or restoration margin to the apical penetration of the probe tip in millimeters; and 3) bleeding on probing (BOP), recorded dichotomously

|| Periopaper, IDE-Interstate, Amityville, NY.

I Periotron 6000, Harco Electronics, Tustin, CA.

\# DiaSorin Inc., Stillwater, MN.

** Cayman Chemical Company, Ann Arbor, MI. 
as 0 or 1 . All clinical measurements were performed after GCF sampling using a manual probe. ${ }^{\dagger \dagger}$ Five examiners (from 2 test centers) performed all clinical measurements and were calibrated prior to the study to ensure adequate intra- and interexaminer reliability. Interexaminer reliability was determined using the intraclass correlation coefficient ${ }^{41}$ to compare an experienced examiner designated as the gold standard to the 5 examiners participating in this study. The intraclass correlation coefficients for probing depth and attachment level measurements ranged from 0.90 to 0.97 , and 0.85 to 0.94 , respectively. Intra-examiner reliability was determined utilizing the percent agreement within $1 \mathrm{~mm}$ between 2 passes and a weighted kappa statistic. The percent agreement within $1 \mathrm{~mm}$ ranged from $95.5 \%$ to $97.3 \%$ and $90.0 \%$ to $95.1 \%$ for probing depth and attachment level measurements, respectively. The weighted kappa statistic ranged from 0.92 to 0.97 and 0.88 to 0.92 for probing depth and attachment level measurements, respectively.

\section{Statistical Analysis}

Mean values for biochemical mediators (ICTP and IL-1) were calculated for each site at all time points (baseline, 1,3 , and 6 months) and averaged within a subject and then across subjects in the 2 groups. Marker levels at each time interval were compacted and analyzed at the subject level using repeated measures analysis of variance (repeated measures ANOVA). Given significance at the 0.05 level, pairwise comparisons were performed using Fisher's protected $t$ tests. Both within-treatment and between-treatment group analyses were performed. Clinical parameters (PD, CAL, and

Table 3. $(\mathrm{pg} /$ site $\pm \mathrm{SE})$

Note: Only deep sites received local therapy. in periodontal patients at all time points $(P<0.01)$.
Table 2.

Subject Group Characteristics at Baseline*

\begin{tabular}{|c|c|c|c|}
\hline & $\begin{array}{l}\text { Healthy Subjects } \\
\qquad(n=8)\end{array}$ & $\begin{array}{l}\text { SRP Plus Vehicle } \\
\qquad(n=25)\end{array}$ & $\begin{array}{l}\text { SRP Plus Minocycline } \\
\qquad(n=23)\end{array}$ \\
\hline $\begin{array}{l}\text { Age in years } \\
\text { (range) }\end{array}$ & $\begin{array}{c}28.7 \\
26.2-34.3\end{array}$ & $\begin{array}{c}45.1 \\
30.9-64.9\end{array}$ & $\begin{array}{c}46.7 \\
34.9-70.4\end{array}$ \\
\hline \% Female & 25 & 60 & 69 \\
\hline \% Smokers & 37.5 & 52.1 & 52.1 \\
\hline \% Bleeding on probing & 12.5 & 72.5 & 77.2 \\
\hline \multicolumn{4}{|c|}{ Probing depth (mean \pm SE) } \\
\hline All sites & $2.10 \pm 0.11$ & $4.28 \pm 0.16$ & $4.42 \pm 0.18$ \\
\hline Shallow sites & N/A & $3.00 \pm 0.11$ & $3.04 \pm 0.10$ \\
\hline Deep sites & $N / A$ & $5.57 \pm 0.14$ & $5.79 \pm 0.19$ \\
\hline \multicolumn{4}{|c|}{ Attachment level (mean \pm SE) } \\
\hline All sites & $1.40 \pm 0.12$ & $4.39 \pm 0.18$ & $4.42 \pm 0.20$ \\
\hline Shallow sites & N/A & $3.17 \pm 0.14$ & $3.13 \pm 0.11$ \\
\hline Deep sites & N/A & $5.61 \pm 0.21$ & $5.71 \pm 0.28$ \\
\hline
\end{tabular}

* No significant differences were noted in demographic or clinical parameters between the vehicle and minocycline treatment groups.

BOP) were compared to ICTP and IL-1 levels utilizing correlation coefficients, which were analyzed for significance using Fisher's $r$ to $\mathrm{z}$ test.

\section{RESULTS}

\section{Subject Characteristics}

Characteristics of the study subjects at baseline are presented in Table 2. Overall, no significant differences

$\dagger \dagger$ PCP-UNC 15, Hu-Friedy Manufacturing Co., Chicago, IL.

ICTP and IL-1 Levels in Deep and Shallow Sites in

Periodontal Patients and Healthy Sites in Control Subjects

\begin{tabular}{|c|c|c|c|c|c|c|}
\hline & \multicolumn{2}{|c|}{$\begin{array}{l}\text { Healthy Subjects* } \\
\qquad(n=8)\end{array}$} & \multicolumn{4}{|c|}{$\begin{array}{l}\text { SRP Plus Vehicle } \\
\quad(n=25)\end{array}$} \\
\hline & \multirow[b]{2}{*}{ ICTP } & \multirow[b]{2}{*}{ IL-I } & \multicolumn{2}{|c|}{ ICTP } & \multicolumn{2}{|c|}{ IL-I } \\
\hline & & & Shallow & Deep & Shallow & Deep \\
\hline Baseline & $27.88 \pm 9.65$ & $1.71 \pm 0.76$ & $154.21 \pm 18.33$ & $207.82 \pm 18.66$ & $36.47 \pm 5.45$ & $60.61 \pm 9.10$ \\
\hline I month & $31.76 \pm 12.92$ & $1.68 \pm 1.09$ & $130.65 \pm 15.49$ & $171.22 \pm 20.03$ & $21.01 \pm 3.60$ & $66.31 \pm 8.36$ \\
\hline 3 months & $32.84 \pm 10.01$ & $1.52 \pm 0.53$ & $119.78 \pm 13.80$ & $172.98 \pm 19.29$ & $31.27 \pm 4.97$ & $60.34 \pm 9.70$ \\
\hline 6 months & $32.69 \pm 10.03$ & $1.54 \pm 0.69$ & $124.26 \pm 15.27$ & $|86.86 \pm 20.7|$ & $32.56 \pm 5.27$ & $56.43 \pm 9.53$ \\
\hline
\end{tabular}

* Significantly lower levels of both markers were found in healthy sites in control subjects than both shallow and deep sites

$\dagger$ Significant reduction from baseline values $(P<0.05)$. 
were noted in demographic or clinical parameters between the minocycline and vehicle treatment groups or between sites within these groups sampled for ICTP and IL-1. Mean ages were 45.1 and 46.7 years for the vehicle and minocycline group, respectively. The percentage of smokers was $52 \%$ in each group. All subjects completed the scheduled examination sessions. A total of 1,536 samples were therefore available for analysis ( 8 sites per subject $\times 48$ subjects $\times 4$ time points), half of which received local therapy (minocycline microspheres or vehicle). The periodontally healthy control group included 6 males and 2 females, with a mean age of 28.7 years. This group was significantly younger than the chronic periodontitis groups $(P<0.05)$. The mean baseline PD and CAL measurements \pm SE at sampled sites were $2.1 \pm 0.11 \mathrm{~mm}$ and $1.4 \pm 0.12$ $\mathrm{mm}$, respectively. There were 3 smokers in the group.

\section{ICTP and IL-1 Levels at Healthy, Shallow, and Deep Sites}

The GCF levels of ICTP and IL-1 in different site strata (i.e., healthy sites in control subjects, and shallow and deep sites in periodontal patients) were compared (Table 3). The healthy control subjects provided 128 sites for analysis ( 4 sites/subject $\times 8$ subjects $\times 4$ time points). A total of 1,536 samples were available for analysis from the periodontitis patients (768 deep sites and 768 shallow sites). Deep sites in periodontal patients contained significantly higher levels of both ICTP and IL-1 than shallow sites in the same patients $(P<0.01)$. Furthermore, both deep and shallow site levels of both markers in periodontal patients were significantly higher than healthy site levels in the control subjects $(P<0.01)$ (Table 3$)$. These differences were statistically significant at baseline and all subsequent

Table 3 (continued).

ICTP and IL-1 Levels in Deep and Shallow Sites in Periodontal Patients and Healthy Sites in Control Subjects (pg/site $\pm \mathrm{SE}$ )

SRP Plus Minocycline $(n=23)$

\begin{tabular}{cccc}
\multicolumn{2}{c}{ ICTP } & \multicolumn{2}{c}{ IL-I } \\
\hline Shallow & Deep & Shallow & Deep \\
\hline $124.45 \pm 16.76$ & $200.44 \pm 24.92$ & $39.84 \pm 8.94$ & $62.20 \pm 8.97$ \\
$113.23 \pm 20.45$ & $132.38 \pm 16.22^{\dagger}$ & $18.01 \pm 3.21$ & $35.54 \pm 5.3 \mid \dagger$ \\
$149.23 \pm 20.12$ & $200.21 \pm 22.98$ & $35.70 \pm 10.95$ & $50.03 \pm 9.93$ \\
$84.29 \pm 12.05$ & $172.27 \pm 19.19$ & $25.81 \pm 7.04$ & $59.79 \pm 10.74$ \\
\hline
\end{tabular}

study time points (i.e. 1, 3, and 6 months), except for ICTP levels between deep and shallow sites in periodontal patients at the 1 -month time point $(P=$ 0.092).

\section{Effects of Treatment on GCF ICTP Levels}

The effects of treatment on the GCF ICTP levels in the 2 study groups are presented in Figure 1. Since only deep sites ( $\geq 5 \mathrm{~mm}$ PD at baseline) were treated with minocycline or vehicle, ICTP levels were stratified by site category (deep versus shallow). In deep sites, statistically significant reductions in ICTP levels were seen only at 1 month in the SRP $+M$ group $(P<0.05)$. ICTP levels were reduced by $33.2 \%$ in the SRP $+M$ group after 1 month, compared to a $17.2 \%$ reduction in the SRP plus vehicle group (Fig. 1). No significant changes in ICTP levels were seen at shallow sites in any of the treatment groups (data not shown).

\section{Effects of Treatment on GCF IL-1 Levels}

The effects of treatment on the GCF IL-1 levels in the 2 study groups are presented in Figure 2. In deep sites, statistically significant reductions in IL-1 levels were noted only at 1 month in the SRP $+M$ treated sites ( $P$ $<0.05)$. Furthermore, at 1 month post-treatment, the SRP $+M$ group demonstrated significantly lower IL-1 levels $(P<0.02)$ compared to the SRP $+\mathrm{V}$ group. This finding is due to a $38.0 \%$ decrease in IL-1 levels 1 month following treatment with SRP $+M$ compared to a 9.4\% increase among patients treated with SRP + V. No significant changes in IL- 1 levels were observed at shallow sites in any of the treatment groups (data not shown).

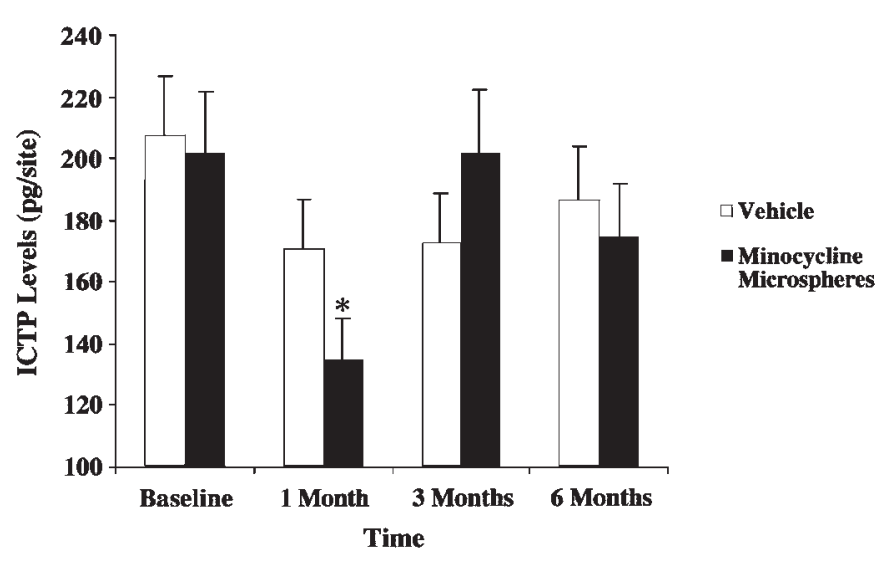

Figure I.

ICTP levels after treatment at periodontal sites receiving local administration of minocycline microspheres. SRP $+M$ was administered to deep sites ( $P D \geq 5 \mathrm{~mm}$ ) in 23 patients, while SRP $+V$ was administered to deep sites in 25 patients. Note potent reductions in GCF ICTP levels at I month in minocycline-treated subjects ( $P$ $<0.05$ ), with minimal effects at 3 and 6 months (NS). *Significant reduction from baseline levels ( $\mathrm{P}<0.05$ ). 


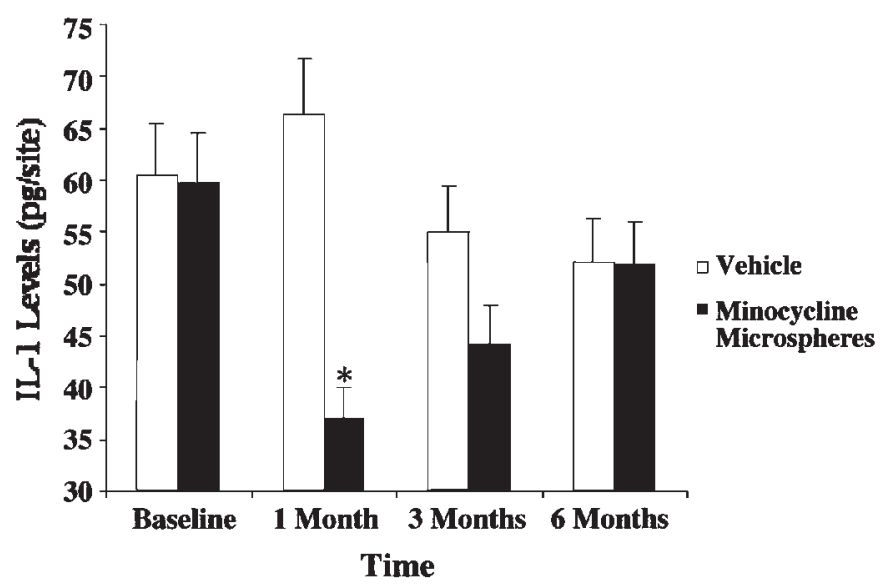

Figure 2.

IL-I levels after treatment at periodontal sites receiving local administration of minocycline microspheres. SRP $+M$ was administered to deep sites ( $P D \geq 5 \mathrm{~mm}$ ) in 23 patients, while $S R P+V$ was administered to deep sites in 25 patients. Note strong reductions in GCF IL-I levels at I month in minocycline-treated patients ( $P$ $<0.05$ ), with minimal effects at 3 and 6 months (NS). At I month, IL-I levels were significantly lower in the SRP $+M$ group compared to the $S R P+V$ group $(P<0.02)$. * Significant reduction from baseline levels $(P<0.05)$ and difference between $S R P+M$ and $S R P+V$ groups at $I$ month $(\mathrm{P}<0.02)$.

\section{Correlation of ICTP and IL-1 Levels With Clinical Parameters}

Correlations among GCF ICTP and IL-1 levels with clinical parameters (PD, CAL, and BOP) were examined. Weak, but statistically significant $(P<0.001)$, correlations were demonstrated between marker levels and all clinical parameters, ranging between 0.13 and 0.37 (data not shown).

\section{DISCUSSION}

Identification of patients or sites with progressive disease or at risk for future periodontal breakdown remains elusive. Similarly, limitations in measurement reliability prevent the accurate assessment of a patient's response to therapy. Host factors in GCF associated with metabolic events responsible for initiation and progression of periodontitis may be useful to identify the current status of a patient and determine individuals and/or sites highly susceptible to disease progression. ${ }^{3}$ The results of this longitudinal study demonstrate that levels of a bone-specific marker (ICTP) and a cytokine associated with bone resorption (IL-1) are higher at sites exhibiting moderate to severe periodontitis compared to sites with less periodontal involvement in periodontitis patients or periodontally healthy individuals. In addition, non-surgical periodontal therapy consisting of mechanical debridement and locally delivered minocycline microspheres provided a statistically significant short-term reduction in IL-1 GCF levels. Therefore, the data sug- gest that markers evaluated in this study may be measures of local tissue destruction and indicators of response to treatment.

The findings of the present study confirm a previous report by Palys et al., ${ }^{21}$ who demonstrated higher levels of ICTP at shallow sites in both gingivitis and periodontitis patients compared to periodontally healthy subjects. In this report, no significant differences were observed in age between the groups. ${ }^{21}$ Thus, it appears that levels of pyridinoline cross-links are not age dependent. Furthermore, Palys et al. noted no significant differences in ICTP levels at sites exhibiting different probing depths among a cohort of treated periodontitis patients. ${ }^{21}$ The findings of both the present study and the Palys et al. report suggest that ICTP levels may be more related to the patient's overall level of periodontal tissue destruction and perhaps their susceptibility to periodontal diseases than to the periodontal anatomy (i.e., probing depth) at a specific site.

Significant reductions were demonstrated 1 month after treatment in GCF ICTP and IL-1 levels only in patients who received SRP plus minocycline microspheres. The reduction in ICTP was nearly twice that achieved by SRP plus vehicle, and the IL-1 reduction was approximately 4 -fold. In addition, at the 1 -month visit, IL-1 levels in patients treated with minocycline microspheres were significantly lower than in patients treated with vehicle. A similar trend was observed for ICTP at 1 month; however, the difference was not statistically significant. Furthermore, in the SRP plus minocycline group, CAL gains of $\geq 2 \mathrm{~mm}$ after 1 month in ICTP sites were associated with a statistically significant concomitant decrease in ICTP levels of 148.1 pg $(55.9 \%)$ from baseline values $(P<0.05)$ (data not shown). The significant improvement noted in biochemical marker levels in the SRP $+M$ group despite the lack of clear differences in response of clinical parameters between the 2 groups suggests that marker levels may provide greater sensitivity than clinical measurements in measuring response to therapy. It is conceivable that changes were present subclinically at the metabolic level that could not be detected by traditional clinical measures. This hypothesis is also supported by the results of correlation testing in which the associations between ICTP and IL- 1 with clinical parameters were relatively weak. As stated by Lamster et al., GCF markers may provide different measures of periodontal disease activity than traditional clinical parameters. ${ }^{42}$ In this regard, while improvements in clinical parameters were maintained over the 6 -month period, GCF levels of ICTP and IL-1 were significantly reduced only at 1 month, but elevated levels returned at 3 and 6 months. The reductions exhibited in ICTP and IL-1 in the minocycline group may be short term and therefore not present 3 months follow- 
ing administration. However, these results may also reflect the need for additional treatment as SRP was provided only at baseline and not at follow-up visits. Additional studies are needed to address whether repeated administration of SRP along with minocycline microspheres is required to achieve long-term reductions in GCF ICTP and IL-1 levels. Further study is also needed to determine whether treatment regimens that reduce these markers are associated with improved patient outcomes.

The observed short-term reductions in ICTP and IL1 levels after treatment with SRP plus minocycline may be related to both the bacteriostatic effect and MMP inhibitory activities of minocycline. ${ }^{43}$ Minocycline $\mathrm{HCl}$ is a tetracycline analog that is active against a broad spectrum of periodontal pathogens. ${ }^{33}$ Previous studies have demonstrated improvements in microbial parameters with the use of locally delivered minocycline compared to debridement and placebo alone. ${ }^{44,45}$ The elevated minocycline concentration achieved with this delivery system may enhance its anti-infective action to penetrate the plaque biofilm compared to systemic administration. In regards to MMP inhibition, it was demonstrated that systemic administration of 40 to 80 $\mathrm{mg}$ of minocycline daily led to a $45 \%$ to $80 \%$ reduction in GCF collagenase activity for at least 2 weeks after initiating treatment. GCF concentrations of minocycline after systemic administration were reported to approximate $10 \mu \mathrm{g} / \mathrm{ml}$. Considering the reported sustained release concentrations of $\geq 340$ $\mu \mathrm{g} / \mathrm{ml}$ for 2 or more weeks achieved with the delivery vehicle utilized in this study, ${ }^{37}$ similar or greater anticollagenolytic activities may have been responsible for the observed reductions in the bone-resorptive markers evaluated. In addition, the known substantive properties of tetracyclines on adhering to the root surface may aid in its extended effects. ${ }^{46}$

Results from this study indicate that GCF ICTP and IL-1 levels are able to capture the short-term effects of treatment with SRP and locally administered minocycline microspheres in chronic periodontitis patients. Further longitudinal studies in larger patient populations are indicated to more thoroughly establish the utility of these markers as adjuncts to evaluate patients with periodontal diseases.

\section{ACKNOWLEDGMENTS}

This study was supported by OraPharma, Inc., Warminster, Pennsylvania.

\section{REFERENCES}

1. Armitage GC. Periodontal diseases: Diagnosis. Ann Periodontol 1996;1:37-215.

2. Haffajee AD, Socransky SS, Lindhe J, Kent RL, Okamoto $\mathrm{H}$, Yoneyama T. Clinical risk indicators for periodontal attachment loss. J Clin Periodontol 1991;18:117-125.

3. Page RC. Host response tests for diagnosing periodon- tal diseases. J Periodontol 1992;63:356-366.

4. Reddy MS. The use of periodontal probes and radiographs in clinical trials of diagnostic tests. Ann Periodontol 1997;2:113-122.

5. Douglass CW, Fox CH. Determining the value of a periodontal diagnostic test. J Periodontol 1991;62:721-730.

6. Goodson JM. Diagnosis of periodontitis by physical measurement: Interpretation from episodic disease hypothesis. J Periodontol 1992;63:373-382.

7. Williams RC, Paquette DW. Periodontal disease diagnosis and treatment: An exciting future. J Dent Educ 1998; 62:871-881.

8. Lamster IB. Evaluation of components of gingival crevicular fluid as diagnostic tests. Ann Periodontol 1997;2: 123-137.

9. Giannobile WV. C-telopeptide pyridinoline cross-links. Sensitive indicators of periodontal tissue destruction. Ann N Y Acad Sci 1999;878:404-412.

10. Calvo MS, Eyre DR, Gundberg CM. Molecular basis and clinical application of biological markers of bone turnover. Endocr Rev 1996;17:333-368.

11. Eyre D. Collagen cross-linking amino acids. Methods Enzymol 1987;144:115-139.

12. Eriksen EF, Charles P, Melsen F, Mosekilde L, Risteli L, Risteli J. Serum markers of type I collagen formation and degradation in metabolic bone disease: Correlation with bone histomorphometry. J Bone Miner Res 1993;8: 127-132.

13. Black D, Marabani M, Sturrock RD, Robins SP. Urinary excretion of the hydroxypyridinium cross links of collagen in patients with rheumatoid arthritis. Ann Rheum Dis 1989;48:641-644.

14. Eastell R, Robins SP, Colwell T, Assiri AM, Riggs BL, Russell RG. Evaluation of bone turnover in type I osteoporosis using biochemical markers specific for both bone formation and bone resorption. Osteoporos Int 1993;3: 255-260.

15. Ulebelhart D, Gineyts E, Chapuy MC, Delmas PD. Urinary excretion of pyridinium crosslinks: A new marker of bone resorption in metabolic bone disease. Bone Miner 1990;8:87-96.

16. Elomaa I, Virkkunen P, Risteli L, Risteli J. Serum concentration of the cross-linked carboxyterminal telopeptide of type I collagen (ICTP) is a useful prognostic indicator in multiple myeloma. Br J Cancer 1992;66:337341.

17. Kylmala T, Tammela TL, Risteli L, Risteli J, Kontturi M, Elomaa I. Type I collagen degradation product (ICTP) gives information about the nature of bone metastases and has prognostic value in prostate cancer. $\mathrm{Br} J \mathrm{Can}$ cer 1995;71:1061-1064.

18. Al-Shammari KF, Giannobile WV, Aldredge WA, et al. Effect of non-surgical periodontal therapy on C-telopeptide pyridinoline cross-links (ICTP) and interleukin-1 levels. J Periodontol 2001;72:1045-1051.

19. Giannobile WV, Lynch SE, Denmark RG, Paquette DW, Fiorellini JP, Williams RC. Crevicular fluid osteocalcin and pyridinoline cross-linked carboxyterminal telopeptide of type I collagen (ICTP) as markers of rapid bone turnover in periodontitis. A pilot study in beagle dogs. $J$ Clin Periodontol 1995;22:903-910.

20. Golub LM, Lee HM, Greenwald RA, et al. A matrix metalloproteinase inhibitor reduces bone-type collagen degradation fragments and specific collagenases in gingival crevicular fluid during adult periodontitis. Inflamm Res 1997;46:310-319.

21. Palys MD, Haffajee AD, Socransky SS, Giannobile WV. 
Relationship between C-telopeptide pyridinoline crosslinks (ICTP) and putative periodontal pathogens in periodontitis. J Clin Periodontol 1998;25:865-871.

22. Talonpoika JT, Hamalainen MM. Type I collagen carboxyterminal telopeptide in human gingival crevicular fluid in different clinical conditions and after periodontal treatment. J Clin Periodontol 1994;21:320-326.

23. Oringer RJ, Palys MD, Iranmanesh A, et al. C-telopeptide pyridinoline cross-links (ICTP) and periodontal pathogens associated with endosseous oral implants. Clin Oral Implants Res 1998;9:365-373.

24. Dewhirst FE, Stashenko PP, Mole JE, Tsurumachi T. Purification and partial sequence of human osteoclastactivating factor: Identity with interleukin-1 beta. $J$ Immunol 1985;135:2562-2568.

25. Page RC. The role of inflammatory mediators in the pathogenesis of periodontal disease. J Periodont Res 1991;26:230-242.

26. Stashenko P, Dewhirst FE, Peros WJ, Kent RL, Ago JE. Synergistic interactions between interleukin 1, tumor necrosis factor and lymphotoxin in bone resorption. $J$ Immunol 1987;138:1464-1468.

27. Kabashima H, Maeda K, Iwamoto Y, et al. Partial characterization of an interleukin-1-like factor in human gingival crevicular fluid from patients with chronic inflammatory periodontal disease. Infect Immun 1990;58: 2621-2627.

28. Lee HJ, Kang IK, Chung CP, Choi SM. The subgingival microflora and gingival crevicular fluid cytokines in refractory periodontitis. J Clin Periodontol 1995;22:885890.

29. Masada MP, Persson R, Kenney JS, Lee SW, Page RC, Allison AC. Measurement of interleukin-1 alpha and -1 beta in gingival crevicular fluid: Implications for the pathogenesis of periodontal disease. J Periodont Res 1990;25:156-163.

30. Reinhardt RA, Masada MP, Johnson GK, DuBois LM, Seymour GJ, Allison AC. IL-1 in gingival crevicular fluid following closed root planing and papillary flap debridement. J Clin Periodontol 1993;20:514-519.

31. Stashenko P, Fujiyoshi P, Obernesser MS, Prostak L, Haffajee AD, Socransky SS. Levels of interleukin-1 beta in tissue from sites of active periodontal disease. J Clin Periodontol 1991;18:548-554.

32. Kinane DF, Winstanley FP, Adonogianaki E, Moughal NA. Bioassay of interleukin-1 (IL-1) in human gingival crevicular fluid during experimental gingivitis. Arch Oral Biol 1992;37:153-156.

33. O'Connor BC, Newman HN, Wilson M. Susceptibility and resistance of plaque bacteria to minocycline. J Periodontol 1990;61:228-233.

34. Golub LM, Ramamurthy N, McNamara TF, et al. Tetracyclines inhibit tissue collagenase activity. A new mechanism in the treatment of periodontal disease. J Periodont Res 1984;19:651-655.

35. Golub LM, Suomalainen K, Sorsa T. Host modulation with tetracyclines and their chemically modified analogues. Curr Opin Dent 1992;2:80-90.

36. Ryan ME, Ramamurthy S, Golub LM. Matrix metalloproteinases and their inhibition in periodontal treatment. Curr Opin Periodontol 1996;3:85-96.

37. Goulding MJ, Sandahl KO, Nowadly CA, Zambon JJ, Christersson LA. Release of minocycline after subgingival deposition by the use of a resorbable polymer. J Periodontol 1991;62:84-85.
38. Williams RC, Paquette DW, Offenbacher S, et al. Treatment of periodontitis by local administration of minocycline microspheres: A controlled trial. J Periodontol 2001;72:1535-1544.

39. Risteli J, Elomaa I, Niemi S, Novamo A, Risteli L. Radioimmunoassay for the pyridinoline cross-linked carboxy-terminal telopeptide of type I collagen: A new serum marker of bone collagen degradation. Clin Chem 1993;39:635-640.

40. Payne JB, Johnson GK, Reinhardt RA, Dyer JK, Maze CA, Dunning DG. Nicotine effects on $\mathrm{PGE}_{2}$ and IL-1 beta release by LPS-treated human monocytes. J Periodont Res 1996;31:99-104.

41. Pihlstrom BL. Measurement of attachment level in clinical trials: Probing methods. J Periodontol 1992;63:10721077.

42. Lamster IB, Holmes LG, Gross KB, et al. The relationship of beta-glucuronidase activity in crevicular fluid to clinical parameters of periodontal disease. Findings from a multicenter study. J Clin Periodontol 1994;21:118-127.

43. Golub LM, Goodson JM, Lee HM, Vidal AM, McNamara TF, Ramamurthy NS. Tetracyclines inhibit tissue collagenases. Effects of ingested low-dose and local delivery systems. J Periodontol 1985;56:93-97.

44. Braswell L, Offenbacher S, Fritz M, Van Dyke TE. Local delivery of Minocin to periodontal lesions in a slow release polymer. J Dent Res 1992;71(Spec. Issue):245(Abstr. 1115).

45. Jones AA, Kornman KS, Newbold DA, Manwell MA. Clinical and microbiological effects of controlled-release locally delivered minocycline in periodontitis. J Periodontol 1994;65:1058-1066.

46. Baker PJ, Evans RT, Coburn RA, Genco RJ. Tetracycline and its derivatives strongly bind to and are released from the tooth surface in active form. J Periodontol 1983;54:580-585.

Correspondence: Dr. William V. Giannobile, Department of Periodontics/Prevention/Geriatrics, University of Michigan, 1011 N. University Avenue, Ann Arbor, MI 48109-1078. Fax: 734/763-5503; e-mail: wgiannob@umich.edu.

Accepted for publication March 8, 2002. 